\title{
Um Cálculo da Espessura da Camada Limite
}

A calculation of the boundary layer thickness

\author{
G. F. Leal Ferreira \\ CP 369, 13560-970, São Carlos, SP
}

Recebido em 15 de janeiro, 2002. Aceito em 1 de fevereiro, 2002.

\begin{abstract}
Revisita-se o problema da camada limite viscosa sobre um perfil plano, derivando-se as equações de Prandtl e depois a de Blasius. Daí, através de procedimento simplificado, uma solução aproximada é obtida cuja precisão ombreia com aquela fornecida pelo conhecido método de von Kármán.
\end{abstract}

The viscous boundary layer problem over a plane is re-visited and the Prandtl system and the Blasius equation are derived. From the latter, a simplified solution is obtained which shows to be close to that generated by the well known von Kármán method.

\section{Introdução}

Em artigo recente nesta revista [1], discutiu-se a questão do perfil de velocidade horizontal na camada limite. Para os leitores menos familiarizados com este conceito, ele foi introduzido por Prandtl no início do século passado [2] para descrever a região de contacto entre um fluido incompressível (=líquido, ou fluido simplesmente) em movimento em relação a um sólido. O líquido adere, por viscosidade, ao sólido a partir da linha de contacto e atinge, na direção normal, a distâncias crescentes - a espessura variável da camada limite -, a velocidade do líquido não perturbada, U. Estamos já imaginando, por simplicidade, que o líquido se desloca em relação a um plano de espessura desprezível em cuja borda de entrada a velocidade é zero. A camada limite é aí também zero, e vai crescendo (direção y) à medida que o líquido avança na direção $\mathrm{x}$. Prandtl [1] admitiu que a velocidade horizontal $\mathrm{u}(\mathrm{x}, \mathrm{y})$ é da forma $U f\left(\frac{y}{\delta(x)}\right)$, em que $\delta(x)$ é a espessura da camada limite em x. A função $f$ satisfaz certas condições de contorno [1], que serão especificadas adiante. $\mathrm{O}$ interesse no presente trabalho é a determinação de $\delta(x)$.

\section{Fluxos horizontal e vertical na camada limite}

Como mencionado acima, o perfil de velocidade horizontal $\mathrm{u}(\mathrm{x}, \mathrm{y})$ é suposto da forma

$$
u(x, y)=U f(\eta)
$$

sendo $\eta=\frac{y}{\delta(x)}=a(x) y$, ou seja, $a(x)=\frac{1}{\delta(x)}$, com $f(0)=0$ e $f(1)=1$.
A Fig. 1 mostra esquematicamente o fluido se aproximando da placa plana, fixa, $\mathrm{P}$, com velocidade uniforme $U$ e atingindo-a em $\mathrm{O}$, a partir de onde se mede a abcissa $x$. A curva (em verdade, superfície) $\mathrm{C}$ delimita a região do fluido fortemente perturbado, contemplado na aproximação, sendo $\delta(x)$, a espessura da camada limite, a grandeza que queremos calcular. No elemento $d x$, a velocidade horizontal do fluido $u(x, y)$ obedece a Eq.1. É interessante notar que para $y$ constante, a velocidade $u$ diminui para $x$ crescente (derivada de $u$ para $y$ constante) e sendo o fluido incompressível, isto significa que há também um movimento vertical, $v(x, y)$, que em $\delta(x)$ atravessa C. Pela equação da continuidade

$$
\frac{\partial u}{\partial x}+\frac{\partial v}{\partial y}=0
$$

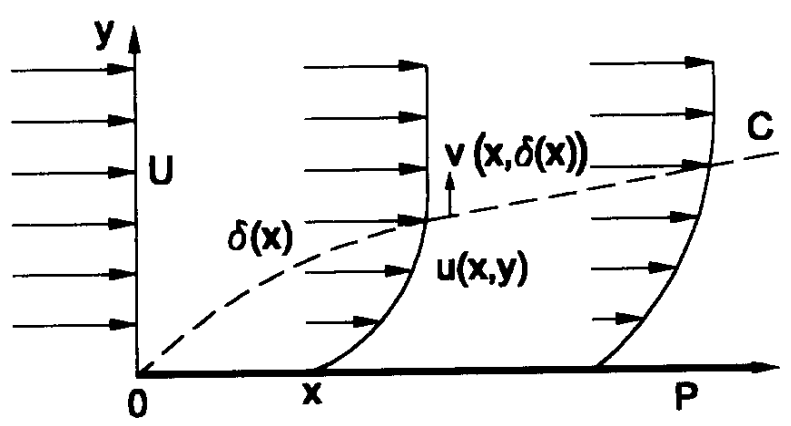

Figura 1. O líquido, com velocidade $U$ atinge a placa OP. A curva $\mathrm{C}$ delimita a camada limite. $u(x, y)$ é a velocidade horizontal e $v(x, \delta(x))$ indica, genericamente, a velocidade vertical ao longo de C.

e da Eq.1

$$
\frac{\partial u}{\partial x}=U y \dot{f} a^{\prime}
$$


em que o ponto significa derivada em relação a $\eta$ e a linha, derivada em relação a $x$. Na maioria dos casos, as variáveis $\eta$ e $x$ não serão mostradas explicitamente. Da Eq.2

$$
\frac{\partial v}{\partial y}=-U y \dot{f} a^{\prime}
$$

oul

$$
\partial v=-U y \dot{f} a^{\prime} \partial y
$$

Mas para $x$ constante, temos $y=\frac{\eta}{a}$ e $\partial y=\frac{d \eta}{a}$ e a Eq.5 integrada dá

$$
\begin{aligned}
v & =-U \frac{a^{\prime}}{a^{2}} \int_{0}^{\eta} \dot{f} \eta d \eta=-U \frac{a^{\prime}}{a^{2}} \int_{0}^{\eta} \eta d f \\
& =-U \frac{a^{\prime}}{a^{2}}\left(\eta f-\int_{0}^{\eta} f d \eta\right)
\end{aligned}
$$

É razoavelmente intuitivo que $v(x, \delta(x))$ deve ser bem menor que $U$ na aproximação proposta. De outra forma, isto é, se $u$ e $v$ fossem da mesma ordem, nenhuma aproximação poderia ser tentada. Supondo-se ser este o caso, e 1) considerando-se os termos no parêntesis da Eq.6 como da ordem da unidade, e 2) que $a(x)=\frac{1}{\delta(x)}$, vê-se que

$$
v \simeq U \delta^{\prime}(x)
$$

e, então, concluímos que

$$
\delta^{\prime}(x)<<1
$$

\section{A Equação de Prandtl}

Tratando-se de escoamento viscoso, devemos usar a Equação de Navier-Stokes. Notemos, porém, que pela Eq.3

$$
\frac{\partial u}{\partial x}=-U \eta \dot{f} \frac{\delta^{\prime}(x)}{\delta(x)}
$$

é da ordem de $U \frac{\delta^{\prime}(x)}{\delta(x)}$, de ordem inferior a $\frac{\partial u}{\partial y}$, que é da ordem de $\frac{U}{\delta(x)}$. Portanto as derivadas horizontais de $u$ podem ser desprezadas em frente às suas derivadas verticais.

Com isso, a Equação de Navier-Stokes (sem gradiente horizontal de pressão) se simplifica, havendo no termo de força viscosa somente a derivada segunda de $u$ em relação a $y$. Ela é

$$
\rho \frac{d u(x(t), y(t))}{d t} \equiv \rho\left(\frac{\partial u}{\partial x} u+\frac{\partial u}{\partial y} v\right)=-\mu \frac{\partial^{2} u}{\partial y^{2}}
$$

sendo $\rho$ a densidade do líquido e $\mu$ a viscosidade. Apesar de $v$ ser bem menor que $u$, os dois termos no parêntesis da Eq.9 são da mesma ordem de grandeza. Isto porque, por compensação, $\frac{\partial u}{\partial x}$ é bem menor que $\frac{\partial u}{\partial y}$. A Eq.9 foi proposta por Prandtl [3].

\section{A Equação de Blasius}

Blasius transformou a Eq.9 numa equação diferencial em $\eta$. Para isto, da Eq.3 temos

$$
\frac{\partial u}{\partial x}=U \eta \dot{f} \frac{a^{\prime}}{a}
$$

Da Eq.1

$$
\frac{\partial u}{\partial y}=U \dot{f} a
$$

$\mathrm{e}$

$$
\frac{\partial^{2} u}{\partial y^{2}}=U \ddot{f} a^{2}
$$

resultando para a Eq.9, $\operatorname{com} \mu_{c}=\mu / \rho$ o coeficiente de viscosidade cinemática,

$$
U^{2} \eta f \dot{f} \frac{a^{\prime}}{a}-U^{2} \frac{a^{\prime}}{a} \dot{f}\left(\eta f-\int_{0}^{\eta} f d \eta\right)=-\mu_{c} U \ddot{f} a^{2}
$$

usando-se as Eqs.1,6,10-12. Notemos que ocorre cancelamento dos dois primeiros termos do lado esquerdo e que

$$
\frac{U a^{\prime}}{\mu_{c} a^{3}}=-\frac{U \delta^{\prime}(x) \delta(x)}{\mu_{c}}=-\frac{U}{2 \mu_{c}} \frac{d \delta^{2}(x)}{d x}=-\frac{1}{K(x)}
$$

em que se definiu $K(x)$ uma função de $x$.Após um certo re-arranjo, a Eq.13 pode ser escrita assim

$$
\frac{\dot{f} \int_{0}^{\eta} f d \eta}{\ddot{f}}=K(x)
$$

de onde se conclui que o lado esquerdo, função de $\eta$, e o lado direito, função de $x$, são iguais a uma constante, que chamaremos de $K$. Com

$$
g(\eta)=\int_{0}^{\eta} f d \eta
$$

a Eq.15 torna-se

$$
\ddot{g} g=K \dddot{g}
$$

equação que Blasius procurou resolver [3]. Na verdade, por uma redefinição de $g$, o fator $K$ na Eq.17 pode ser eliminado. Tomaremos, porém, aqui um outro rumo mais simples, procurando determinar $K$ de uma forma aproximada pela Eq.17 e retornando depois à Eq.14 para a determinação de $\delta(x)$. 


\section{Determinação aproximada de $K$ e de $\delta(x)$}

Vamos determinar $K$ impondo um perfil aproximado para $f(\eta)$.Para a solução exata, a Eq.17 é satisfeita para todos os valores de $\eta, 0<\eta<1$. Com um perfil aproximado, vamos impor que a igualdade entre o lado esquerdo e direito da Eq.17 se dê em média, ou seja

$$
\int_{0}^{1}(\ddot{g} g-K \dddot{g}) d \eta=0
$$

No tratamento usual do problema devido a von Kármán, no qual se iguala a perda de momento do líquido com o atrito viscoso sobre a placa, usa-se o perfil proposto por Prandtl, dado por

$$
f(\eta)=\frac{1}{2}\left(3 \eta-\eta^{3}\right)
$$

que satisfaz as condições especificadas abaixo da Eq.1 (isto é, $f(0)=0$ e $f(1)=1$ ) e, adicionalmente, $\dot{f}(1)=0$, ou seja, a velocidade horizontal, Eq.1, atinge suavemente o valor $U$ em C. Das Eqs.16 e 19 obtêm-se

$$
\begin{gathered}
g=\frac{1}{2}\left(\frac{3}{2} \eta^{2}-\frac{1}{4} \eta^{4}\right) \\
\ddot{g}=\frac{3}{2}\left(1-\eta^{2}\right) \\
\dddot{g}=-3 \eta
\end{gathered}
$$

e substituindo-se as Eqs.20-22 na Eq.16, obtém-se

$$
K=\frac{13}{140}
$$

e retornando-se à Eq.14, tem-se agora

$$
\frac{d \delta^{2}(x)}{d x}=\frac{280 \mu_{c}}{13 U}
$$

ou seja,

$$
\delta(x)=4,64 \sqrt{\frac{\mu_{c} x}{U}}
$$

O fato de $\delta(x)$ ser inversamente proporcional a $U$ e a $\rho$,este através de $\mu_{c}$, mostra que as forças inerciais têm um papel preponderante na formação da camada, ou seja, uma maior densidade de momento do líquido impõe camada limite mais fina. A força viscosa sobre a placa deve ser, grosso modo, diretamante proporcional a $U$ e inversamente proporcional a $\delta(x)$. Vemos, então, que a força de arraste sobre a placa, de acordo com Eq.25, vai $\operatorname{com} U^{\frac{3}{2}}$.

\section{Comentários}

A solução de von Kármán [2] leva ao coeficiente numérico 4,65 na Eq.25 (não sabemos se ela é matematicamente equivalente àquela dada acima e a diferença, 4,65 e 4,64 adviriam de aproximações diferentes). Por outro lado, a de Blasius, a partir da equação diferencial da Eq.17 [3], por solução em série, dá o valor convencionado de 4,52 .

Mesmo para escoamentos turbulentos, há formação de camada limite viscosa na região próxima de $x=0$ [2]. Tomando-se escoamento de água, com $\mu_{c} \simeq 1,2^{-2}$ $\mathrm{cm}^{2} / \mathrm{s}$, com velocidade de $1 \mathrm{~m} / \mathrm{s}$, para a qual já há turbulência, a Eq.25 dá para $\delta(x) \approx 5^{-2} \sqrt{x} \mathrm{~cm}$, isto é, uma camada bem estreita.

O que aconteceria se usássemos para o perfil de velocidades $f(\eta)$, Eq.19, um polinômio de ordem superior a três, mantendo-se as mesmas três condições de contorno abaixo da Eq.19, como determinado em [1]? Evidentemente, o perfil dependeria agora de um parâmetro extra, $b, f(\eta, b)$. Obtidos, então, os correspondentes $g$, $\dot{g}, \ddot{g}, \dddot{g}$, a Eq.18 forneceria uma função, vamos dizer, $H(K, b)=0$. Impondo-se agora um mínimo (na verdade um extremo) para $H(K, b)$, isto é, $\partial H / \partial b=0$, $K$ poderá ser determinado. Quer dizer, a eventual arbitrariedade na definição do perfil pode ser eliminada 'dinamicamente ' pela Eq.18 e imposição de um mínimo em relação aos parâmetros excedententes.

Por fim, vamos testar se a condição de validade da aproximação na Eq.7' de fato se verifica. Temos

$$
\delta^{\prime}(x)=2,33 \sqrt{\frac{\mu_{c}}{U x}}<<1
$$

mostrando que para pontos próximos à borda de entrada (ponto O na Fig.1) a aproximação não é boa. Para o caso do escoamento considerado acima, isto ocorreria para $x<<6,5 x 10^{-4} \mathrm{~cm}$. Como a perturbação causada pela própria borda não foi considerada, isto parece não prejudicar muito a solução, que seria válida para $x$ suficientemente grande, eventualmente transicionando para a camada limite turbulenta, para $x$ ainda maiores [2].

\section{Agradecimentos}

$\mathrm{O}$ autor agradece ao $\mathrm{CNPq}$ a bolsa de produtividade.

\section{Referências}

[1] Katia B. de Lacerda e A. E. A. Amorim, Rev. Brasil. Ensino Física, textbf23, 196 (2001).

[2] Victor L. Streeter, Mecânica dos Fluidos, Editora McGraw-Hill do Brasil; Ltda.,1974,Cap.V.

[3] J. W. Daily e D. R. F. Harleman, Fluid Dynamics, Addison-Wesley Publ. Co. Inc., 1973, Cap.10. 